\title{
Perfeccionamiento editorial de publicaciones científicas universitarias. E1 caso de la revista Centro Azúcar
}

\begin{abstract}
Editorial improvement of university scientific publications. The case of Centro Azúcar Journal
\end{abstract}

\author{
Luis Ernesto Paz Enrique \\ luisernestope@uclv.cu \\ Universidad Central “Marta Abreu” de Las Villas, Cuba. \\ Merlyn González Mas \\ mgmas@uclv.cu \\ Universidad Central “Marta Abreu” de Las Villas, Cuba. \\ Lauren Reyis Canto Hernández \\ 1canto@uclv.cu \\ Universidad Central "Marta Abreu" de Las Villas, Cuba.
}

\section{Resumen}

Los estudios de vigilancia favorecen la identificación de las últimas tendencias de un objeto para la toma de decisiones. La revista Centro Azúcar no tiene referentes del comportamiento de su perfil temático a nivel internacional. Se adolece de un estudio de vigilancia científica que facilite información relativa a las tendencias de la temática que publica. Lo anterior determinó que los objetivos del presente estudio estuvieran encaminados a: 1) caracterizar la producción científica de la Revista Centro Azúcar entre el 2015 al 2017 y 2) examinar la producción científica internacional sobre la temática: Caña de Azúcar en la Web de la Ciencia durante los años 2015 y 2017. Para la obtención de resultados se aplicaron métodos en los niveles teórico y empírico, fundamentalmente el método bibliométrico. Como principales resultados se obtuvieron que la Revista Centro Azúcar cuenta con una productividad científica caracterizada por una amplia presencia de coautores y miembros del consejo 
editorial/científico de la publicación. El aspecto mencionado con anterioridad afecta la calidad científica de la publicación, condicionado por la de endogamia evidente y la ausencia de autores expertos. Los resultados obtenidos ofrecen información relevante sobre la producción de la temática e identifican a Brasil como un alto productor. Se aspira que con los resultados obtenidos se reformulen las políticas de la revista Centro Azúcar para favorecer su posicionamiento en bases de datos de prestigio.

\title{
Palabras clave
}

Revistas científicas; Caña de azúcar; Revista Centro Azúcar; Método biobliométrico; Vigilancia de la ciencia.

\begin{abstract}
Surveillance studies favor the identification of the latest trends of an object for decision making. Centro Azúcar Journal does not have references of the behavior of its thematic profile at an international level. It suffers from a scientific surveillance study that provides information on the trends of the subject it publishes. The foregoing determined that the objectives of the present study were aimed at: 1) characterizing the scientific production of the Centro Azúcar Journal between 2015 and 2017 and 2) examining the international scientific production on the subject: Sugar Cane in the Web of Science during the years 2015 and 2017. To obtain results, methods were applied at the theoretical and empirical levels, fundamentally the bibliometric method. The main results were that Centro Azúcar Magazine has a scientific productivity characterized by a wide presence of co-authors and members of the editorial board. The previously mentioned aspect affects scientific publication quality, conditioned by the evident endogamy and ausence of expert authors. The previously mentioned aspect affects scientific publication quality, conditioned by the evident endogamy oand the absence of expert authors. The results obtained offer relevant information on the production of the subject and identify Brazil as a high producer. It is hoped that with the results obtained, the policies of the Centro Azúcar Journal will be reformulated, to favor its positioning in prestigious databases.
\end{abstract}

\section{Keywords}

Scientific journals, Sugarcane, Centro Azúcar Journal, Biobliometric Method, Science Surveillance. 
Recibido: 6/03/2019

Aceptado: $12 / 05 / 2019$

DOI: https://dx.doi.org/10.5557/IIMEI10-N18-029051

Descripción propuesta: Paz Enrique, Luis Ernesto; González Mas, Merlyn; Canto Hernández, Lauren Reyis 2019. Perfeccionamiento editorial de publicaciones científicas universitarias. El caso de la revista Centro Azúcar. Métodos de Información, 10(18), 29-51

\section{Introducción}

Actualmente las revistas científicas Junto a la función social de difundir la ciencia, cumplen otros importantes roles (Fernández 2004): son el medio más rápido para conocer los últimos avances sobre un campo específico del conocimiento, constituyen el mecanismo propicio para evaluar la actividad científica, permiten controlar y certificar la calidad de los resultados de investigación (es decir su exactitud y novedad) y si se garantiza su accesibilidad, se transforman en un archivo público del conocimiento. Son consideradas como los eslabones básicos en el proceso de transferencia y difusión de la ciencia y un instrumento privilegiado para la comunicación entre los investigadores (López y Cordero 2005).

La bibliometría es la disciplina encargada de analizar repertorios bibliográficos para el apoyo de la toma de decisiones. Uno de los principales usos de la bibliometría es el análisis de revistas científicas para diagnosticar su calidad. Las revistas científicas constituyen en la actualidad la principal vía de socialización de los resultados de investigación. El criterio anterior está determinado porque "la principal vía de comunicación formal de los resultados de la investigación científica continúa siendo el artículo científico publicado en revistas arbitradas" (Sarduy et al. 2014). Además de que "uno de los componentes fundamentales de la ciencia y la actividad científica son las publicaciones" (Paz et al. 2015). En Iberoamérica la principal fuente de información para los investigadores es el acceso a los paper (artículos científicos publicados) como para acceder a los resultados publicados de 
primera mano. Las revistas científicas son el medio con mayor prestigio social para los investigadores.

Los estudios de vigilancia científica apoyados en la Bibliometría, favorecen la obtención de datos estadísticos a los cuales se somete a valoración para la toma de decisiones, por lo que juegan un papel esencial. El uso más frecuente de la Bibliometría es el análisis de publicaciones seriadas (Arencibia et al. 2014), (Paz, Hernández y Garcés 2016). Es una herramienta ampliamente usada, constituye un método de investigación para evaluar el desempeño de la investigación (Durieux y Gevenois 2010). El análisis de producción científica favorece la formulación de políticas para la actuación de las temáticas de publicación de las publicaciones seriadas. La producción científica es un componente medular de la cultura de la sociedad y un elemento esencial en el proceso de generación y evaluación (Silva,2012).

Los análisis de la producción científica generalmente se apoyan de bases de datos que indizan revistas y publicaciones científicas. Varios estudios emplean indicadores bibliométricos para describir la producción científica de una temática o área específica, tal es el caso de las investigaciones desarrolladas por Flores y de Andrade (2015); Franco et al. (2014); Anchondo et al. (2014); Murce et al. (2013); Paula et al. (2013); Peinado y Reis (2014); Zacca et al. (2015).

Las revistas científicas universitarias muestran el quehacer investigativo de una institución que, entre otras funciones, destina recursos y potencia las investigaciones para aportar conocimientos. La Universidad Central "Marta Abreu" de Las Villas posee múltiples publicaciones periódicas. Bajo la editorial Feijóo se encuentran: ISLAS, para las ciencias sociales y humanísticas; Centro Agrícola, para las ciencias agropecuarias, Centro Azúcar para las ciencias químicas. Se localiza en este centro además la revista: Biotecnología Vegetal editada por el Centro de Biotecnología de las Plantas (IBP), la Revista Varela de ciencias pedagógicas y la Revista Ciencia y Actividad Física de la facultad de la enseñanza del deporte.

La revista Centro Azúcar tiene una frecuencia trimestral. Pertenece a la Facultad de Química y Farmacia de la Universidad Central "Marta Abreu" de Las Villas. Fue fundada en 1973. Surgió para publicar artículos científicos 
sobre la producción de azúcar, pero ha ampliado su perfil a otros temas afines. Tiene número de ISSN electrónico 2223-4861. Es de acceso abierto y sus artículos son arbitrados a ciegas por especialistas de gran experiencia y prestigio. Las temáticas de publicación de la revista son:

- Industria de la caña de azúcar

- Derivados de la caña de azúcar

- Obtención de biocombustibles

- Problemas técnicos, económicos, ambientales, sociales de operación y desarrollo de la caña de azúcar

- Procesos químicos en general

- Derivados de la caña de azúcar

- Simulación y optimización de procesos

- Gestión ambiental

- Desarrollo de procesos para productos farmacéuticos

- Análisis químico de productos

- Tópicos misceláneos relacionados con la Ingeniería Química

Los artículos publicados en esta revista son indizados o resumidos por: DOAJ, Bibliat: Bibliografía Latinoamericana Periódica, CubaCiencias, Informe Académico, Fuente Académica, Fuente Académica Premier y EBSCOhost. Desde el año 2016 la revista fue indizada en la colección SciELO luego de varias revisiones e intentos por ser incluida en la base de datos mencionada. La revista está financiada por: el Ministerio de Educación Superior de Cuba-MES, Universidad Central "Marta Abreu" de las VillasUCLV y la Facultad de Química y Farmacia.

La revista Centro Azúcar no tiene referentes del comportamiento de su perfil temático a nivel internacional. Se adolece de un estudio de vigilancia científica que facilite información relativa a las tendencias de la temática que publica. Esto determina que los objetivos del presente estudio estén encaminados a: 1) caracterizar la producción científica de la Revista Centro Azúcar entre el 2015 al 2017 y 2) examinar la producción científica internacional sobre la temática: Caña de Azúcar en la Web de la Ciencia (Web of Science: WOS) durante los años 2015 y 2017. 
Existen gran cantidad de revistas de impacto que favorece la investigación de la Caña de Azúcar. La vigilancia del comportamiento a nivel internacional de la producción científica de esta temática servirá de guía para el perfeccionamiento de la labor editorial de la revista Centro Azúcar en cuanto a su posicionamiento por bases de datos de prestigio que le permita mejorar su visibilidad. Para el logro de este fin es necesaria una actualización de sus temáticas de publicación. Los beneficiarios del estudio serán los editores y los usuarios o lectores de la revista al tener un producto más actualizado acorde a las tendencias actuales de las temáticas afines a la revista. La investigación favorecerá el posicionamiento de la publicación de igual forma que la misma se mantendría en la base de datos SciELO. Se identifica como antecedente principal del estudio el desarrollado por Paz, et al. (2016) donde se analizan la calidad de la Revista Centro Azúcar desde una perspectiva métrica.

\section{Materiales y métodos}

El estudio presentado se clasifica como investigación descriptiva, ya que no se modifica realidad, sino que se describe. Es longitudinal-retrospectiva, analiza el período desde 2015 a 2017. Se utilizan métodos en los niveles teórico y empírico. En el nivel teórico se emplearon el analítico sintético, inductivo deductivo y sistémico estructural. En el nivel empírico se empleó el análisis documental clásico y el método bibliométrico. Los pasos seguidos para el desarrollo del método bibliométrico se describen a continuación:

- Confección de la base de datos: se confeccionaron dos bases de datos. La primera contenía la producción científica de la Revista Centro Azúcar entre los años 2015 y 2017. Fue importada al EndNote X7 desde la base de datos SciELO Citation Index de la WOS. La segunda base de datos fue importada del Science Citation Index (SCI) y las especializadas en ciencias químicas: Index Chemicus y Current Chemical Reactions de la WOS.

- Dimensión temporal del estudio: el estudio abarcó el período de 2013 al 2015. El período seleccionado corresponde a la inclusión de la Revista Centro Azúcar en SciELO. 
- Se empleó como estrategia de búsqueda criterios bilingües, definiendo a la temática fundamental Caña de Azúcar (Sugar Cane). Se almacenaron los registros en una base de datos EndNote X 7.

- Normalización y procesamiento de los datos: en la primera base de datos se obtuvieron un total de 93 registros correspondientes a la producción de la revista en el período analizado. Del estudio de vigilancia de la temática caña de azúcar se recuperaron un total de 1705 registros, de los cuáles 31 no presentaron autoría para un 1,8\% de la muestra.

- Herramientas utilizadas: para la obtención de rankings y para el cálculo de los indicadores seleccionados se empleó el programa Microsoft Excel, del paquete de programas Microsoft Office 2007, donde fueron creadas las tablas y gráficos correspondientes. Se empleó además el software VOSviewer para la visualización de resultados.

Indicadores escogidos: Los indicadores seleccionados para el análisis de la producción científica de la revista se muestran en la Tabla 1 y para la vigilancia de la temática caña de azúcar en la Tabla 2.

\begin{tabular}{|c|c|}
\hline Variable & Indicador \\
\hline Año & - Producción científica por años \\
\hline Autor & $\begin{array}{l}\text { - Autores más productores de comunicaciones } \\
\text { científicas } \\
\text { - Índice de transitoriedad }\end{array}$ \\
\hline Colaboración & $\begin{array}{l}\text { - Índice de coautoría } \\
\text { - Coautoría por años } \\
\text { - Coautoría total } \\
\text { Tipos de colaboración }\end{array}$ \\
\hline Impacto & $\begin{array}{l}\text { Número de citas } \\
\text { Número de documentos citados } \\
\text { Por ciento de documentos citados }\end{array}$ \\
\hline Palabras clave & $\begin{array}{l}\text { Palabras clave más productivas } \\
\text { Palabras clave más productivas por años }\end{array}$ \\
\hline Resumen & Términos más frecuentes en los resúmenes \\
\hline
\end{tabular}

Tabla 1: Variables e indicadores escogidos para describir la producción cientifica de la Revista Centro Azúcar entre el 2013 y el 2015 (Fuente: elaboración propia). 


\begin{tabular}{|c|c|}
\hline Variable & Indicador \\
\hline Autor & - Autores más productores de comunicaciones científicas \\
\hline País & - Países más productores de comunicaciones científicas \\
\hline Insti & $\begin{array}{l}\text { - Instituciones más productoras de comunicaciones } \\
\text { científicas } \\
\text { - Principales entidades financiadoras }\end{array}$ \\
\hline Revista & - Revistas más productivas de comunicaciones científicas \\
\hline
\end{tabular}

Tabla 2: Variables e indicadores escogidos para describir la producción científica de la temática Caña de Azúcar entre el 2013 y el 2015 (Fuente: elaboración propia).

La técnica empleada fue el análisis documental clásico que permitió la consulta de fuentes y estudios similares.

\section{Resultados y discusión}

\section{Análisis del estudio bibliométrico de la productividad científica de la Revista Centro Azúcar en el período 2015-2017}

La producción de artículos por años en la Revista Centro Azúcar muestra una diferencia significativa en el 2017. La producción se muestra en la Ilustración

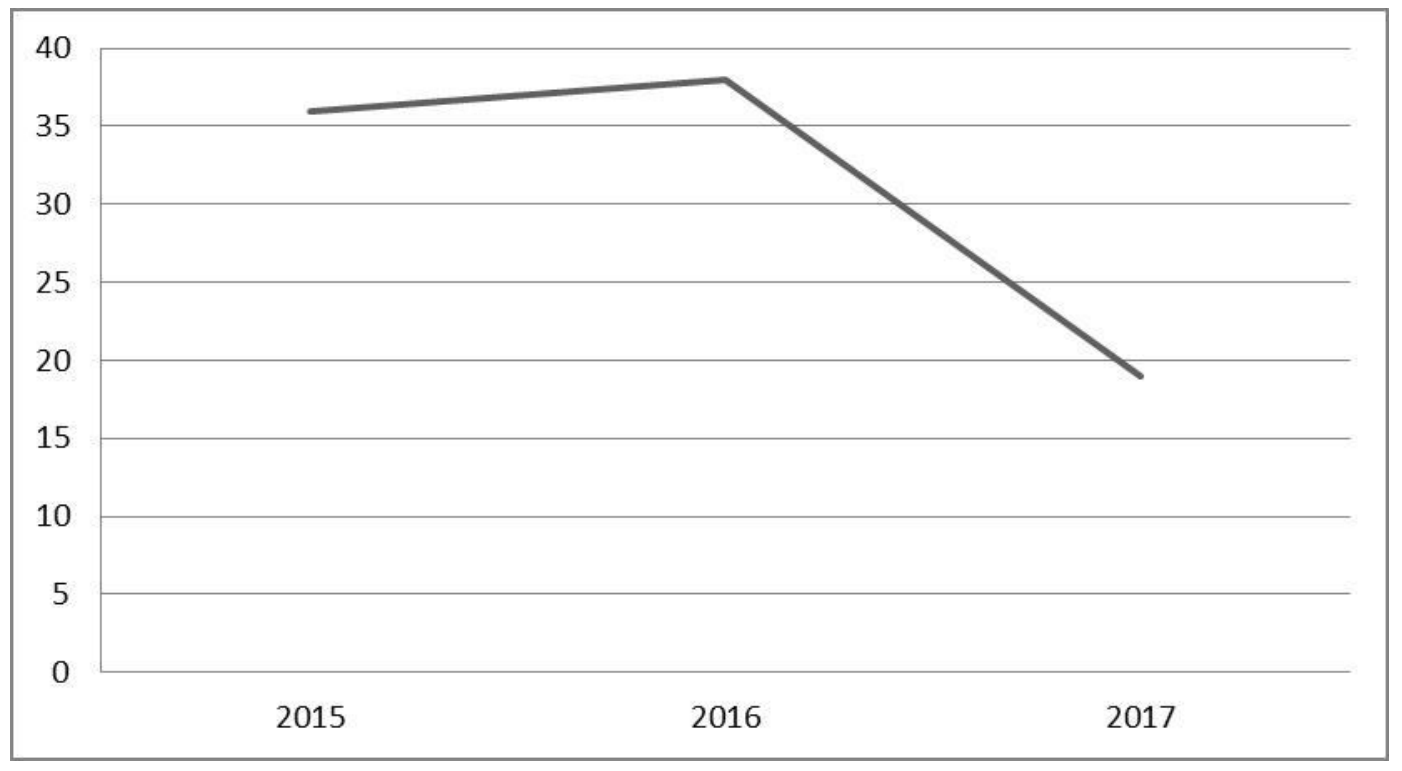

Ilustración 1: Producción cientifica de la Revista Centro Azúcar en el periodo 2015-2017 (Fuente: elaboración propia). 
El año más productivo el 2016 con 38 artículos lo que representa un 40,9\% y como año menos productivo el 2017 con un total de 19 artículos que representa un 20,4\%. Este indicador refleja que es necesario trabajar para que aumente el número de publicaciones ya que se evidencia una tendencia a disminuir. Las revistas científicas deben publicar una cantidad de artículos de forma estable en los números. La disminución en el año 2017 representa que hubo un descenso de alrededor del 50\% en cuanto a productividad. Atendiendo al impacto se analizaron la cantidad de citas recibidas por años, los resultados se muestran en la Tabla 3.

\begin{tabular}{|l|l|l|l|l|}
\hline Años & $\begin{array}{l}\text { Número de } \\
\text { documentos }\end{array}$ & $\begin{array}{l}\text { Número de } \\
\text { citas }\end{array}$ & $\begin{array}{l}\text { Número de } \\
\text { documentos citados }\end{array}$ & $\begin{array}{l}\text { Por ciento de } \\
\text { documentos citados }\end{array}$ \\
\hline 2015 & 36 & 3 & 3 & 8,33 \\
\hline 2016 & 38 & 2 & 2 & 5,26 \\
\hline 2017 & 19 & 0 & 0 & 0 \\
\hline Total & $\mathbf{9 3}$ & $\mathbf{5}$ & $\mathbf{5}$ & $\mathbf{5 , 3 7}$ \\
\hline
\end{tabular}

Tabla 3: Impacto de la Revista Centro Azúcar en el periodo 2015-2017 (Fuente: elaboración propia).

Los resultados obtenidos muestran que en el período se evidencia poco impacto de las investigaciones publicadas en la revista. En este período solo se citaron 5 artículos, una sola vez cada uno. Debe destacarse que por lo general deben pasar dos años de la publicación de los artículos para que aparezcan las primeras citas. En el caso anterior se ubica el 2017 que en el momento en que se realizaba la presente investigación no había obtenido citas. El impacto debe potenciarse a partir de la actualización de las temáticas de investigación de la revista. Lo anterior supone que la comunidad científica muestre interés en investigaciones de pertinencia.

La Revista Centro Azúcar presenta altos índices de coautoría, representando a más de 3 autores por artículos. Para el análisis de los resultados obtenidos en cuanto a la coautoría se realiza una distribución de la de artículos publicados por años y la cantidad de autores que estos contenían. Los resultados de la coautoría y transitoriedad por años y total se muestran en la Tabla 4. 


\begin{tabular}{|l|l|l|l|l|l|}
\hline Años & $\begin{array}{l}\text { Cantidad } \\
\text { de artículos }\end{array}$ & $\begin{array}{l}\text { Cantidad de } \\
\text { autores }\end{array}$ & $\begin{array}{l}\text { Autores } \\
\text { transitorios }\end{array}$ & $\begin{array}{l}\text { Índice de } \\
\text { coautoría }\end{array}$ & $\begin{array}{l}\text { Índice de } \\
\text { transitoriedad }\end{array}$ \\
\hline 2015 & 36 & 142 & 99 & 3,94 & $69,51 \%$ \\
\hline 2016 & 38 & 145 & 101 & 3,81 & $69,65 \%$ \\
\hline 2017 & 19 & 75 & 75 & 3,94 & $100 \%$ \\
\hline Total & $\mathbf{9 3}$ & $\mathbf{3 6 2}$ & $\mathbf{2 7 5}$ & $\mathbf{3 , 8 9}$ & $\mathbf{7 5 , 9 6 \%}$ \\
\hline
\end{tabular}

Tabla 4: Índice de transitoriedad, indice de coautoría, coautoría por años y coautoría total (Fuente: elaboración propia).

Se visualiza en la tabla que la tendencia de publicar entre dos o más autores se cumple con un comportamiento medio a partir de los altos índices de transitoriedad (75,96 \% son autores transitorios). La coautoría oscila entre los índices 3,81 y 3,94. El resultado no es favorable, pues aunque los investigadores tengan una alta productividad, los altos índices de coautoría reflejan la existencia de pocos especialistas al identificarse muchos autores en los artículos. Los resultados coinciden con los de Paz et al. (2016), los autores mencionados agredan que por lo general en las investigaciones sobre química aplicada los autores no publican solos, las investigaciones se desarrollan con el apoyo de técnicos y otros especialistas que desarrollan estudios prácticos en laboratorios.

La coautoría es una de las formas más tangibles y bien documentadas de colaboración científica (Glänzel y Schubert 2004). Los científicos colaboran para ganar visibilidad, reputación, habilidades complementarias y acceso a recursos. Estos factores se relacionan con la organización social e intelectual de la comunidad científica, y no con factores históricos, de proximidad geográfica o del modelo económico centro-periferia (Leydesdorff y Wagner 2009).

Las relaciones de colaboración de los autores que publican en la Revista Centro Azúcar se clasifican de media. Según Ayala et al. (2012) los colaboradores medianos son aquellos que han publicado entre 2 y 8 trabajos en colaboración. Esto influye cuando hay pocos especialistas en la disciplina y por tanto los autores tienen que reunirse para construir un artículo (Paz et al., 2016). La colaboración está dada por publicaciones que tienen contribución de más de un autor. Los tipos de colaboración realizada en la Revista Centro Azúcar se muestran en la Tabla 5. 


\begin{tabular}{|l|l|}
\hline Tipos de colaboración & Número de documentos \\
\hline Colaboración nacional & 65 \\
\hline Colaboración internacional & 31 \\
\hline No colaboración & 6 \\
\hline Colaboración itrainstitucional (UCLV) & 15 \\
\hline
\end{tabular}

Tabla 5: Tipos de colaboración cientifica de la Revista Centro Aquicar en el periodo 2015-2017 (Fuente: elaboración propia).

De las 93 publicaciones 15 pertenecen en su totalidad autores de la Universidad Centra "Marta Abreu" de Las Villas. En el período se han hecho colaboraciones nacionales con varias universidades del país como la Universidad de Cienfuegos, la Universidad de Camagüey, la Universidad de Guantánamo, la Universidad de Sancti Spíritus, la Universidad de Pinar del Río y la Universidad Tecnológica de La Habana José Antonio Echeverría. Otras instituciones nacionales firmantes de artículos son la Empresa de Cementos Cienfuegos, la Estación Provincial de Investigaciones de la Caña de Azúcar, la Fábrica de Cementos Cienfuegos, el Instituto Cubano de Investigaciones de los Derivados de la Caña de Azúcar, la Oficina Nacional de Inspección Estatal, el Instituto Cubano de Investigaciones de los Derivados de la Caña de Azúcar, la Empresa Comercializadora de Servicios Técnicos y Tecnológicos, la Planta Bioprocesos, la Refinería de petróleo Camilo Cienfuegos, el Centro de Estudios Ambientales de Cienfuegos, el Centro de Investigaciones del Petróleo, la Consultoría Jurídica de Aguada de Pasajeros, la Corporación Cuba Ron S.A, la Delegación Territorial CITMA, la Empresa Azucarera 5 de Septiembre y la Empresa de Aprovechamiento Hidráulico de Villa Clara.

Se emplearon solamente dos idiomas: el inglés y el español. El idioma más favorecido fue el español con un total de 89 artículos. El resultado obtenido es algo esperado en consonancia al idioma oficial de la publicación. Se la ausencia de traducciones de las normas editoriales, aspecto que pudiera determinar la colaboración y la poca presencia de otros idiomas. El español representa el $95.5 \%$ del total de artículos publicados. Atendiendo a la productividad por autores se identificaron 824 autores en los 180 artículos. Los autores más productivos se observan en la Tabla 6. 


\begin{tabular}{|l|l|l|l|}
\hline Autores más productivos & Cantidad de artículos & Autor principal & Coautor \\
\hline Erenio González Suárez & 11 & 0 & 11 \\
\hline Meilyn González Cortés & 6 & 4 & 2 \\
\hline Juan Esteban Miño Valdés & 5 & 1 & 4 \\
\hline Irenia Gallardo Aguilar & 5 & 0 & 5 \\
\hline Rubén Espinosa Pedraja & 4 & 0 & 4 \\
\hline
\end{tabular}

Tabla 6: Autores más productivos en la Revista Centro Azúcar en el período 2015-2017 (Fuente: elaboración propia).

Se destaca a González-Suárez, Erenio con un total de 11 artículos publicados, de ellos solo dos como autor principal. El autor mencionado labora dentro de la revista como editor y revisor. Lo anterior provoca que se eleve grandemente los índices de endogamia editorial en la publicación que puede traer graves consecuencias como la no aceptación de la publicación para su indización en bases de datos de prestigio. González-Suárez, Erenio pertenece al Departamento de Ingeniería Química, de la Facultad de Química y Farmacia de la UCLV. El resto de los autores también pertenecen a la UCLV y están vinculados profesionalmente a la revista, lo anterior muestra una endogamia editorial evidente.

Las actuales temáticas de la revista se evidencian en las palabras clave con mayor producción por años, las mismas se muestran en la Ilustración 2. 


\section{Productividad de palabras clave por años}

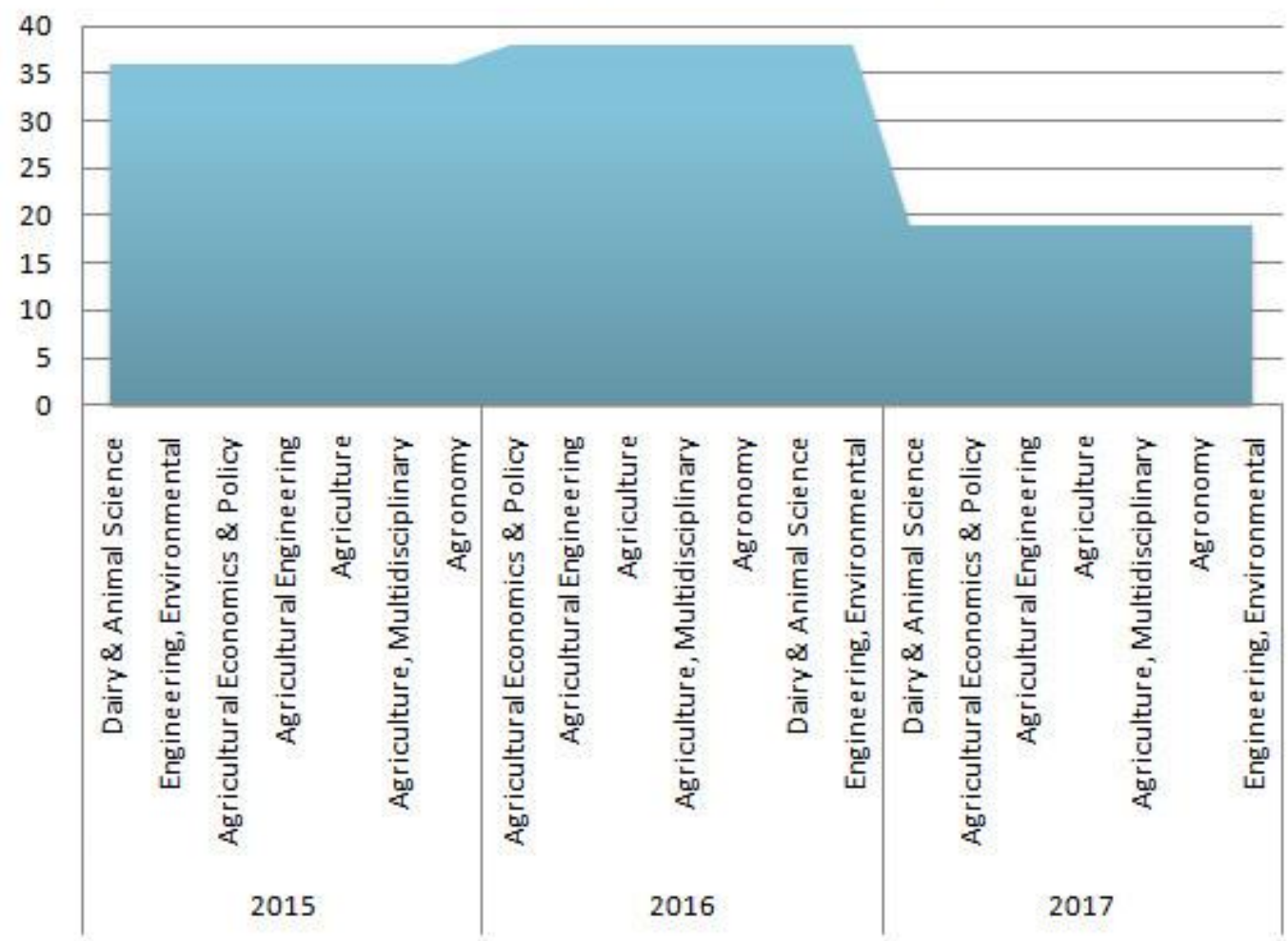

Ilustración 2: Palabras clave más productivas por años (Fuente: elaboración propia).

Se evidencia una coincidencia de palabras clave en todos los años analizados. Los términos refieren a las actuales temáticas de publicación de la revista y fundamentalmente a los aspectos que se relacionan con procesos químicos a partir de la caña de azúcar. Existen dentro de la UCLV una serie de instituciones cuyas investigaciones tributan a las áreas de investigación de la Revista Centro Azúcar. Las principales instituciones son: Departamento de Ingeniería Química, Departamento de Licenciatura en Química, Departamento de Farmacia, Centro de Bioactivos Químicos, Centro de Investigaciones de Química Aplicada, Centro de Biotecnología de las Plantas, Departamento de Ciencia Agropecuarias, Departamento de Ingeniería Agrícola.

Las palabras clave más productivas se observan en la Tabla 7.

\begin{tabular}{|l|l|}
\hline Palabras clave & Frecuencia \\
\hline Dairy \& Animal Science & 93 \\
\hline Agricultural Economics \& Policy & 93 \\
\hline
\end{tabular}




\begin{tabular}{|l|l|} 
Agricultural Engineering & 93 \\
\hline Agriculture & 93 \\
\hline Agriculture, Multidisciplinary & 93 \\
\hline Agronomy & 93 \\
\hline Engineering, Environmental & 93 \\
\hline Etanol & 6 \\
\hline Bagazo & 5 \\
\hline
\end{tabular}

Tabla 7: Palabras clave más productivas en la Revista Centro Azúcar en el perído 2015-2018 (Fuente: elaboración propia).

En la tabla aparecen dos palabras clave que, aunque no cuentan con la amplia del resto; si están directamente vinculadas con la revista. Ethanol y bagazo son términos frecuentes en la investigación de la caña de azúcar. Los términos mencionados pueden clasificarse como subproductos de la caña de azúcar. Se realizó un gráfico de calor a partir de la densidad de las palabras más frecuentes en los resúmenes de los artículos publicados. Los resultados se observan en la Ilustración 3.

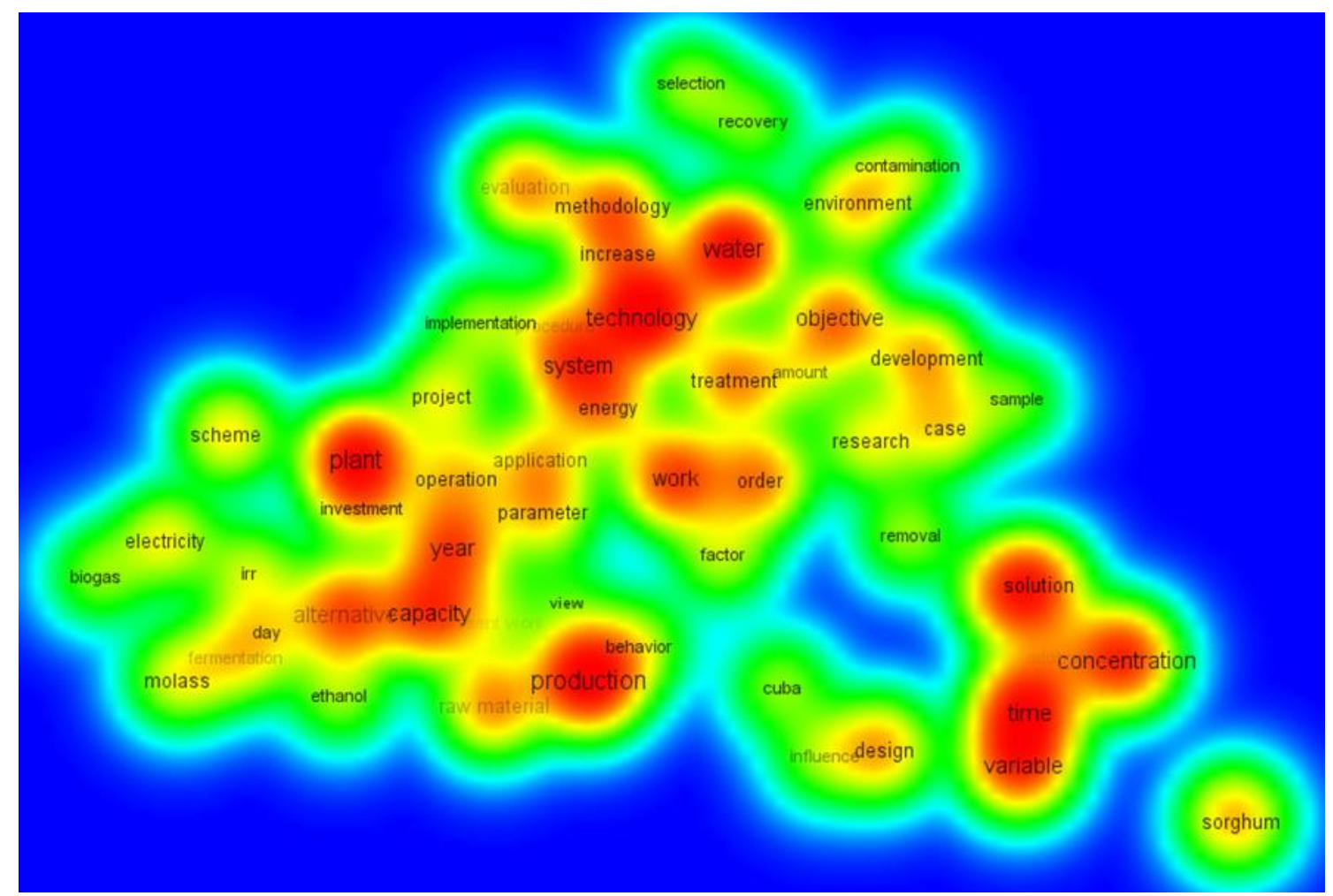

Ilustración 3: términos más frecuentes en los resúmenes de los artículos publicados por la Revista Centro Azúcar en el período 2015-2018 (Fuente: elaboración propia). 
Se destacan las palabras tiempo (time), solución (solution), tecnología (technology) y producción (production); las mismas están relacionadas con los procesos químicos. En el caso de agua (water), fermentación (fermentation), biogas y tratamiento (treatment); se corresponden con las investigaciones desarrolladas por el Centro de Bioactivos Químicos. El centro mencionado tiene una alta presencia en la productividad de la Revista Centro Azúcar. El CBQ es una organización dedicada a la investigación de producción de sustancias químicas que presentan actividad biológica. El centro obtiene el Furfural a partir de los desechos de la caña de azúcar y a través de una hidrólisis ácida, teniendo como resultado la obtención de Ingredientes Farmacéuticos Activos (IFA). Estos resultados están comprometidos con la línea de desarrollo de la industria farmacéutica y biotecnológica del programa estratégico de desarrollo a nivel nacional (Paz et al. 2015).

Los objetivos del centro de investigación reflejan un papel protagónico de acuerdo a la actividad científica, el desarrollo, producción y comercialización de productos con acción biológica. Los compuestos obtenidos por síntesis química, son utilizados en la salud humana, veterinaria y en la esfera agrícola, así como subproductos del proceso fabril generando beneficios económicos. El CBQ ejecuta proyectos de colaboración, servicios de investigacióndesarrollo, asistencia técnica con financiamiento, pagos por licencia y donaciones, exportación de productos farmacéuticos y formación académica de postgrado.

\section{Estudio de vigilancia de la temática caña de azúcar en la Web Of Science (WoS)}

Los autores más productivos son Detmann, E. y Glaz, B. con 14 registros cada uno. En la Tabla 8 se muestran los autores más productivos.

\begin{tabular}{|l|l|}
\hline Autores & Registros \\
\hline Detmann, E. & 14 \\
\hline Glaz, B. & 14 \\
\hline Comstock, J.C. & 12 \\
\hline Edme, S.J. & 12 \\
\hline Pires, A.J.V. & 12 \\
\hline Davidson, R.W. & 11 \\
\hline
\end{tabular}




\begin{tabular}{|l|l|}
\hline Doherty, W.O.S. & 11 \\
\hline Gilbert, R.A. & 11 \\
\hline Glynn, N.C. & 11 \\
\hline Sood, S. & 11 \\
\hline
\end{tabular}

Tabla 8: Autores más productivos (Fuente: elaboración propia).

Los más productivos se corresponden con investigadores localizados en universidades de múltiples territorios. Los autores identificados en los primeros lugares con un total de 12 artículos son de nacionalidad brasileña y estadounidense respectivamente. Dentro de los autores más productivos existe un predominio de aquellos con nacionalidad estadounidense, registrándose siete que proceden de este país. Todos los investigadores firmantes de comunicaciones científicas proceden del Estado de la Florida. La institución más representada fue la USDA-ARS Sugarcane Field Station con los investigadores: Glaz, B.; Comstock, J.C.; Edme, S.J.; Davidson, R.W.; Gilbert, R.A.; Glynn, N.C. y Sood, S. Los autores mencionados están asociados, en su mayoría, a la Universidad de la Florida. Del resto de los investigadores Detmann, E. y Pires, A.J.V. firman como investigadores de la Universidad Federal de Viçosa y están asociados a la entidad financiadora del Conselho Nacional de Desenvolvimento Cientifico e Tecnologico (CNPq). El autor Doherty, W.O.S. es de nacionalidad australiana.

La nación más productora de comunicaciones científicas es Brasil con 619. Dicho país es reconocido como el mayor productor de caña de azúcar a nivel internacional, por lo que no es casuístico este resultado. Varios estudios destacan el protagonismo de esta nación en el sector de la producción azucarera, entre ellos destacan (Alonso y Arcilla, 2014), (Puerto y Sauera, 2015), (Samuel, et al., 2014). En segundo lugar se localiza Estados Unidos con 194 registros seguido de la India con 132. Los lugares por naciones se muestran en la Tabla 9.

\begin{tabular}{|l|l|}
\hline Países/Territorios & Registros \\
\hline Brasil & 619 \\
\hline Estados Unidos & 194 \\
\hline India & 132 \\
\hline República Popular China & 84 \\
\hline Australia & 81 \\
\hline
\end{tabular}




\begin{tabular}{|l|l|}
\hline México & 58 \\
\hline Alemania & 53 \\
\hline Francia & 47 \\
\hline Inglaterra & 43 \\
\hline
\end{tabular}

Tabla 9: Países más productivos (Fuente: elaboración propia).

La producción de artículos científicos e investigaciones relacionados con la temática Caña de Azúcar se encuentra encabezado por Brasil, representando el $36.3 \%$ de la producción total. En segundo lugar, se encuentra Estados Unidos cuya producción representa un $11.3 \%$ del total. La producción de Brasil triplica la producción de la nación que ocupa la segunda posición. El hecho de que Brasil y la India ocupen el primer y tercer lugar respectivamente y China en cuarta posición no es un hecho casuístico, esto responde a un proyecto nacional de desarrollo de estas 3 economías emergentes que facilita la inversión de capitales para el desarrollo de la ciencia bajo el precepto de I + $\mathrm{D}+\mathrm{i}$ (Investigación + Desarrollo + Innovación). Las principales instituciones productoras de comunicaciones científicas se muestran en la Tabla 10.

\begin{tabular}{|l|l|}
\hline Instituciones / Organizaciones & Registros \\
\hline Universidade de Sao Paulo & 129 \\
\hline Universidade Estadual Paulista & 110 \\
\hline Universidade Estadual de Campinas & 59 \\
\hline Universidade Federal de Vicosa & 55 \\
\hline United States Department of Agriculture USDA & 42 \\
\hline Universidade Federal do Rio de Janeiro & 41 \\
\hline Universidade Federal de Lavras & 30 \\
\hline Empresa Brasileira de Pesquisa Agropecuaria Embrapa & 28 \\
\hline Universidade Federal de Sao Carlos & 28 \\
\hline Florida State University System & 26 \\
\hline
\end{tabular}

Tabla 10: Instituciones más productivas (Fuente: elaboración propia).

Del total de 10 instituciones productoras 8 son de origen brasileño, representando el $80 \%$ de las entidades identificadas. Los primeros cuatro lugares son ocupados por instituciones del territorio brasileño y del total se identifican 8 universidades. Los datos obtenidos demuestran el desarrollo de la investigación desde la academia, teniendo un gran peso en la productividad el desarrollo de estudios en programas de postgrado y proyectos de 
investigación. Se identifica solamente una empresa (de origen brasileño) y una organización gubernamental (Estados Unidos).

Las instituciones más productoras se ubican en los países más productivos. En el caso de los Estados Unidos, este resultado es producto del financiamiento de las investigaciones científicas de una economía desarrollada. El criterio anterior favorece que se desarrollen estudios sobre la temática, esencialmente en centros de investigación y universidades de Lousiana y La Florida donde se concentra la principal producción de caña de azúcar de este país.

La principal entidad financiadora es Conselho Nacional de Desenvolvimento Cientifico e Tecnologico (CNPq) con un registro de 116 comunicaciones científicas, esta cifra ubica a la entidad financiadora CNPq en una supremacía absoluta con respecto a las demás que se registran. El segundo, tercer y cuarto lugar lo ocupan: Coordenacao de Aperfeicoamento de Pessoal Dd Nivel Superior (CAPES), Fundacao de Amparo a Pesquisa do Estado de Sao Paulo (FAPESP), Fundacao de Amparo a Pesquisa do Estado de Minas Gerais (FAPEMIG) y National Natural Science Foundation of China (NSFC) con registros de 69, 66 y 26 respectivamente. Las entidades financiadoras tienen una representación en un total de registros tal y como se representan en la tabla 11.

\begin{tabular}{|l|l|}
\hline Entidades Financiadoras & Registros \\
\hline CNPQ & 116 \\
\hline CAPES & 69 \\
\hline FAPESP & 66 \\
\hline FAPEMIG & 26 \\
\hline NSFC & 26 \\
\hline
\end{tabular}

Tabla 11: Entidades financiadoras más productivas (Fuente: elaboración propia).

Con la excepción de NSFC el resto de las entidades financiadoras son de origen brasileño. Las entidades financiadoras identificadas ofrecen y financian proyectos de investigación en múltiples áreas del conocimiento. El hecho que cuatro de las entidades pertenezcan a Brasil ratifica y afianza el desarrollo científico que ha alcanzado el país latinoamericano en los últimos años. Actualmente la nación oferta programas maestrías y doctorados potenciando el desarrollo de la ciencia. 
La revista más productiva identificada en el estudio es International Sugar Journal con un total de 114 artículos identificados en el período seleccionado. El segundo lugar lo ocupa la Brazilian Journal of Animal Science con 61 y en tercer lugar aparece v con 54 registros. En la Tabla 12 se observan las revistas más productivas establecidas en un ranking descendente.

\begin{tabular}{|l|l|}
\hline Títulos de fuentes & Registros \\
\hline International Sugar Journal & 114 \\
\hline Brazilian Journal of Animal Science & 61 \\
\hline Sugar Tech & 54 \\
\hline Bioresource Technology & 50 \\
\hline Semina Ciencias Agrarias & 29 \\
\hline Biomass Bioenergy & 22 \\
\hline Journal of Agricultural and Food Chemistry & 22 \\
\hline Industrial Crops and Products & 21 \\
\hline Revista Brasileira de Ciencia do Solo & 20 \\
\hline Quimica Nova & 19 \\
\hline Listy Cukrovarnicke a Reparske & 17 \\
\hline Journal of Plant Registrations & 16 \\
\hline Bioscience Journal & 15 \\
\hline
\end{tabular}

Tabla 12: Revistas más productivas (Fuente: elaboración propia).

Para comprender la relación de las publicaciones se emplea el modelo matemático de Bradford. Se toman todos los registros de cada una de las revistas o títulos de fuentes identificados. Se suman todos los artículos y se obtiene un total de 1707, este resultado es dividido entre tres ya que son las áreas que se desean representar y se obtiene como resultado 569 artículos por zonas aproximadamente. Se coloca en cada área valores entre 538 y 605 artículos con el objetivo de ponderar cada una. Se representan los resultados en un gráfico que muestra la zona núcleo y las demás zonas. La representación del modelo puede observarse en la Ilustración 4. 


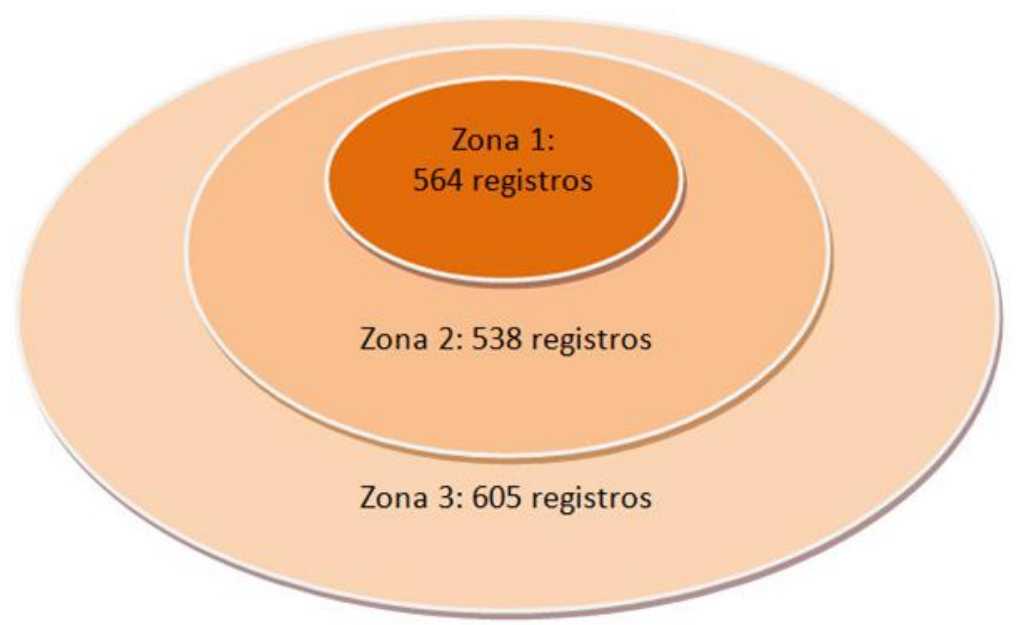

Ilustración 4: Modelo de Bradford aplicado para mostrar la dispersión de producción cientifica de la temática Caña de Azúcar (Fuente: elaboración propia).

La zona 1 o núcleo cuenta con 564 artículos, ocupa desde los registros de 114 hasta 12 y se corresponden con las revistas núcleo sumando un total de 21 . Se establece esta zona debido a que, aunque el valor no alcanza la cifra de 569; si se adicionara los valores siguientes excedería en 61 al resultado definido a representar. La zona 2 cuenta con 538 artículos perteneciente a los valores de 11 hasta 3 artículos, estos pertenecen a un total de 113 revistas científicas. La zona 3 o periférica cuenta con 605 artículos, la misma representa todos los autores que tienen dos artículos o son autores transitorios (productividad de 1 registro), esta zona comprende un total de 507 revistas.

\section{Conclusiones}

La Revista Centro Azúcar cuenta con una productividad científica caracterizada por una amplia presencia de coautores y miembros del consejo editorial/científico de la publicación. El aspecto mencionado con anterioridad afecta la calidad científica de la publicación, condicionado por la presencia de endogamia evidente y la ausencia de autores expertos.

Se destaca que en el año 2017 hubo una disminución de artículos publicados de casi el 50\%, aspecto que debe ser mejorado por parte de la Revista Centro Azúcar. La publicación debe hacer traducciones de su política editorial para potenciar la productividad en otros idiomas. 
La temática Caña de Azúcar ha sido abordada desde diversas áreas del saber. Las tendencias de la producción científica en esta temática se centra en estudios realizados desde la academia con el protagonismo de universidades brasileñas.

La nación brasileña ocupa los primeros lugares cono nación, instituciones y entidades financiadores mayores productoras de comunicaciones científicas. Los resultados obtenidos ratifican y afianza el desarrollo científico que ha alcanzado el país latinoamericano en los últimos años. La mayor parte de la producción de Brasil se identifica que se origina desde las universidades, esto está dado por la gran oferta programas maestrías y doctorados de la nación potenciando el desarrollo de la ciencia.

Las entidades financiadoras que mayor producción científica tienen sobre la temática Caña de Azúcar radican en su mayoría en Brasil. El resultado está en correspondencia a un amplio plan de financiamiento que sostiene esta nación para el desarrollo de investigaciones, especialmente en programas de postgrado.

Existe gran cantidad de revistas de impacto que favorece la investigación de la Caña de Azúcar. Se identifica que en los principales países productores de caña de azúcar se localizan las entidades financiadoras fundamentales de comunicaciones científicas. De igual forma en estas naciones se encuentran las revistas más productivas, hecho esperado atendiendo a la actividad y producción científica descrita sobre estas regiones.

\section{Bibliografía}

ALONSO, J. y ARCILA, A., 2014. Eficiencia semifuerte del mercado internacional del azúcar entre los años 2001 y 2011. Cuadernos de Economía, 33(62), 145-161.

ANCHONDO, R., TARANGO, J. y ASCENSIO, G., 2014. Características de los sujetos-objetos en la producción científica de química avanzada: el caso del CIMAV, México. IBERSID, 8(2014), 143-147. 
ARENCIBIA, D.F., BETANCOURT, V., GONZÁLEZ, N., PUIG, Y., BIART, O., FERNÁNDEZ, D.F. y OCHOA, R., 2014. Estudio bibliométrico de la producción científica de VacciMonitor (2000-2013). VacciMonitor, 23(2), 41-48.

CÓRDOVA, S., ARÉVALO, R.A., SANOMYA, R.M., BERTONCINI, E.I., ARÉVALO, L.U., CHAILA, S. y FUSTAINO, M.L., 2014. Saccharum spp. en Brasil. Una revisión. Avances en Investigación Agropecuaria, 18(3), 49-64.

DURIEUX, V. y GEVENOIS, P.A., 2010. Bibliometric indicators: quality measurements of scientific publication. Radiology, 2(255), 342-351.

FERNÁNDEZ, E. (2014). Revistas cientificas electrónicas: estado del arte. Digital-CSIC. San José: e.Revistas.

FLORES, V. y DE ANDRADE, T., 2015. Comportamento de cidadania organizacional: caracterização da produção científica internacional no período de 2002 a 2012. Revista de Administracao Mackeñie, 2(6), 45-71.

FRANCO, A.M., SANZ, V.J., WANDEN, J. y MELIAN, L., 2014. La producción científica iberoamericana en ciencias de la nutrición: La indización en PubMed y Google Scholar. Nutrición Hospitalaria, 5(30), 1165-1172.

GLÄNZEL, W. y SCHUBERT, A., 2004. Analysing scientific networks through coauthorship. Netherlands: Kluwer Academic Publishers.

LEYDESDORFF, L. y WAGNER, C., 2009. Is the United States losing ground in Science?: a global perspective on the world science system. Scientometrics, 78(2009), 23-36

LÓPEZ, M. y CORDERO, G., 2005. Un intento por definir las características generales de las revistas académicas electrónicas. Razón y Palabra, 43(2005), 131.

MURCE, P.P., COELHO, F.A., REZENDE, R., PASCHOAL, T. y DA SILVA, A.I., 2013. A produção científica brasileira sobre a gestão de recursos humanos entre 2001 e 2010. Revista de Administracao Mackenzie, 4(15), 110-134.

PAULA, C.C., CABRAI, I.E., SOUZA, L.E., BRUM, C.N., SILVA, C.B. у PADOIN, S.M., 2013. HIV/AIDS in childhood and adolescence. Trends in Brazilian cientific production. Investigación \& Educación en Enfermería, 2(31), 277 286.

PAZ, L.E., HERNÁNDEZ, E.A. y GARCÉS, R., 2015. Análisis de citas para la descripción de la calidad de las publicaciones científicas: El caso de la Revista Latina de Comunicación Social. Serie Bibliotecología y Gestión de Información, 95(2015), 1-38. 
PAZ, L.E., CÉSPEDES, A. y HERNÁNDEZ, E.A., 2016. Análisis métrico de las comunicaciones de la Revista Centro Azúcar. Biblios, 62(2016), 17-33.

PAZ, L.E., ROMERO, R. y HERNÁNDEZ, E.A., 2015. Productividad científica del Centro de Bioactivos Químicos en el período 2008-2012. Avanzada Cientifica, 18(3), 1-17.

PEINADO, J. y REIS, A.A., 2014. Produção científica em gestão de operações no brasil: uma análise de temas, autores e instituições de pesquisa no período entre 2001 e 2010. Revista de Administracao Mackenzie, 5(15), 224-255.

PUERTO, J.A. y SAUERA, I.L., 2015. Política energética en Brasil: Implementación del componente renovable. Energética, 45(2015), 95-106.

SARDUY, Y., LLANUSA, S.B., URRA, P. y ANTELO, J.M., 2014. Caracterización de la producción científica de la Escuela Nacional de Salud Pública referenciada en la base de datos Scopus, 2006-2012. Educación Médica Superior, 2(28), 243-259.

SILVA, L.C., 2013. El índice-H y Google Académico: una simbiosis cienciométrica inclusiva. Acimed, 2(23), 308-322.

ZACCA, G., CHINCHILLA, Z., VARGAS, B. y DE MOYA, F., 2015. Patrones de comunicación e impacto de la producción científica cubana en salud pública. Revista Cubana de Salud Pública, 2(41), 200-216. 